\title{
Parálisis facial en otitis media: Revisión bibliográfica
}

\author{
Facial paralysis in otitis media. Literature review
}

Michel Royer F, Carlos Stott C', Mª Paz Rivas².

\begin{abstract}
RESUMEN
La parálisis facial secundaria a una otitis media es una conocida complicación de infecciones agudas y crónicas. Su fisiopatología es desconocida pero la presión elevada en el oído medio, la osteítis, la invasión bacteriana y la neurotoxicidad, podrían estar involucradas. Los exámenes de laboratorio no entregan mayor información para determinar la etiología de la parálisis facial y la tomografía computada de oídos sería el estudio radiológico de elección ya que permite identificar el compromiso óseo, la extensión de la enfermedad y posibles alteraciones anatómicas existentes, además de ser utilizado para el diagnóstico de eventuales complicaciones intracraneales. $B$ tratamiento de la parálisis en el curso de una otitis media aguda es médico, con antibióticos y esteroides, con buen pronóstico de recuperación; mientras en una otitis media crónica el tratamiento es quirúrgico y el pronóstico global peor.
\end{abstract}

Palabras clave: Parálisis facial, otitis media, colesteatoma

\begin{abstract}
Facial paralysis secondary to otitis media is a well known complication of acute and chronic infection. The physiopathology of facial nerve paralysis secondary to otitis media of not known, but high middle ear pressure, osteitis, direct bacterial invasion and neurotoxicity could be involved. Laboratory studies have failed to provide clues as to determining the etiology of facial nerve palsy. Ear CT is the radiologic study of choice, as it allows the identification of bone destruction, disease extension and possible anatomic alterations, and may also be used as a screening for intracranial complications. Acute otitis media treatment is medical with antibiotics and steroids, with good prognosis, whereas in chronic otitis media the treatment is surgical, with worst global prognosis.
\end{abstract}

Key words: Facial paralysis, otitis media, cholesteatoma.

2 Interna de Medicina, Facultad de Medicina. Universidad de Chile. 


\section{INTRODUCCIÓN}

La otitis media es una patología común bien conocida en la práctica médica, cuyas complicaciones tanto intracraneales como extracraneales han disminuido considerablemente gracias a la introducción de la terapia antibiótica'. La inflamación del oído medio tiende a limitarse en la caja timpánica y mastoides, pero en algunas ocasiones el proceso puede afectar estructuras relacionadas, como el canal óseo del nervio facial causando parálisis facial. En la era preantibiótica este compromiso fue una complicación frecuente de la otitis media, con una incidencia estimada de $0,5 \%$ a $0,7 \%$. Actualmente se ha vuelto un fenómeno excepcional y su incidencia se ha reducido al $0,005 \%$ en la evolución de una otitis media aguda $(\mathrm{OMA})^{2,3}$. En otitis media crónica (OMC) la frecuencia alcanza de $0,16 \%$ a $5,1 \%$ y es mayor en países en desarrollo 4 .

La etiopatogenia de la parálisis facial como complicación de la otitis media es poco conocida, sin embargo se han descrito varios mecanismos fisiopatológicos que podrían estar relacionados con la génesis de ésta.

Esta complicación constituye una emergencia médico quirúrgica, por lo que es de suma importancia su diagnóstico oportuno para iniciar un tratamiento adecuado. Las conductas terapéuticas son adoptadas en forma precoz con la finalidad de obtener un mejor resultado funcional, pero la base de estas decisiones aún no presentan una evidencia científica concluyente.

\section{ANATOMÍA}

日 nervio facial, que corresponde al séptimo par craneano, es un nervio mixto que posee fibras motoras que inervan los músculos de la expresión facial, del estribo, posauriculares, estilohioideo y el vientre posterior del digástrico; fibras parasimpáticas para las glándulas submandibular, sublingual y lagrimal; fibras sensoriales que inervan los dos tercios anteriores de la lengua; y también ramos aferentes sensitivos de la piel del conducto auditivo externo.

日 núcleo motor del facial se encuentra en la protuberancia, recibe inervación desde el giro precentral de la corteza motora y entrega fibras hacia la región facial ipsilateral y en forma bilateral para el tercio superior del rostro. Las fibras parasimpáticas provienen del núcleo salival superior, se dirigen a las glándulas lacrimal, nasal y palatinas a través del nervio petroso superficial mayor, y a las glándulas submandibular y sublingual através de la cuerda del tímpano. Las fibras del gusto de los dos tercios anteriores de la lengua alcanzan el ganglio geniculado mediante la cuerda del tímpano.

Una vez que el nervio facial ingresa al conducto auditivo interno sigue un tortuoso camino en su canal óseo, conocido como canal facial o de Falopio, hasta su emergencia en el agujero estilomastoídeo. Cásicamente se describen cuatro segmentos:

1. La porción meatal de $8 \mathrm{~mm}$ a $10 \mathrm{~mm}$ que se ubica en el conducto auditivo interno, en una posición superior al nervio coclear y anterior al nervio vestibular superior.

2. La porción laberíntica de $2 \mathrm{~mm}$ a $4 \mathrm{~mm}$ abarca desde el meato auditivo hasta el ganglio geniculado, donde se emiten los nervios petrosos superficiales mayor y menor.

3. E segmento timpánico, de aproximadamente $11 \mathrm{~mm}$, se inicia en la primera curvatura (de $\left.40^{\circ} \mathrm{a} 80^{\circ}\right)$, tiene un curso anteroposterior en la pared medial de la caja timpánica, en una posición superior a la apófisis cocleariforme (del músculo del martillo) y luego inferior al canal semicircular lateral, manteniéndose superior a la ventana oval y platina del estribo, emite un ramo para el músculo del estribo y alcanza la segunda rodilla.

4) 日 segmento mastoideo se inicia tras la segunda curvatura (en $90^{\circ}$ ), tiene una longitud aproxima da de $12 \mathrm{~mm}$ a $14 \mathrm{~mm}$ y emite la cuerda del tímpano para finalizar en el orificio estilomastoideo.

Tras su emergencia de la base del cráneo, el nervio facial atraviesa la glándula parótida y se subdivide para inervar los músculos de la cara

Debe recordarse que el segmento timpánico del nervio facial es vulnerable a los procesos inflamatorios del oído medio ya que la pared ósea en ese segmento es delgada y frecuentemente presenta dehiscencias, que corresponden a peque ños espacios no patológicos en el espesor de la pared del canal. La frecuencia estimada de 
dehiscencias es variable de acuerdo a los pacientes incluidos en las series y la metodología utilizada. Estudios clínicos, con diagnóstico durante un evento quirúrgico, describen una prevalencia de $6 \%$ a $35 \% \%^{6-8}$, mientras estudios anatómicos las demuestran desde $19 \%$ a $57 \% \%^{7-10}$. Se encuentran principalmente cercanas a la ventana oval ${ }^{9,11,12}$ y su principal causa se produciría durante la osificación del canal, proceso que puede afectarse por alteraciones del cartílago de Reichert ${ }^{9,12}$. También son más frecuentes producto de cirugías otológicas previas, inflamación persistente con osteítis y pre sencia de colesteatomas ${ }^{11,13}$.

\section{FISIOPATOLOGÍA}

Al momento de producirse un daño neural, de acuerdo a la magnitud de éste y las estructuras comprometidas, se pueden presentar cambios tanto proximales como distales al sitio de la noxa, lo que determinan las alteraciones futuras y la potencial recuperación de la integridad anatómica y funcional. Estas alteraciones han sido clasificadas en tres grupos:

1. Neuropraxia, cuando se presenta daño en la vaina nerviosa, pero sin alteraciones en el axón, por lo que la conducción nerviosa se altera en el sitio de la lesión, pero está conservada hacia proximal y distal, siendo su recuperación habitualmente completa.

2. Axonotmesis, cuando se produce una alteración de la vaina nerviosa y el axón, con preservación del endoneuro, generándose daño a distal (degeneración walleriana), pero manteniendo una potencial recuperación.

3. Neurotmesis, cuando existe daño tanto en axón como sus estructuras conectivas, con degenera ción walleriana, produciéndose a futuro reinervación sólo parcial y con alteraciones evidentes.

\section{FISIOPATOLOGÍA DE PARÁLISIS FACIAL EN OTITIS MEDIA}

Dada su estrecha relación anatómica con el oído y el hueso temporal, el nervio facial se encuentra expuesto a diferentes noxas en este compartimiento (traumáticas, inflamatorias, autoinmunes, etc.).
La fisiopatología de la parálisis facial en otitis media no se encuentra completamente establecida, siendo probable que más de un factor influya en su aparición, tales como la elevación de la presión del oído medio, la osteítis o la inflamación aguda, que podrían afectar directamente el nervio facial y favorecer su disfunción.

\section{Presión elevada en el oído medio}

日 nervio facial ocupa entre el $35 \%$ a $65 \%$ del canal de Falopio, mientras el resto corresponde a tejido conectivo y vascular ${ }^{14}$, por lo que la presencia de edema inflamatorio puede generar compresión y trombosis de vasa nervorum con isquemia neural secundaria Esta disfunción del nervio tras su compresión e isquemia ha sido descrita en modelos animales, en los cuales la parálisis inducida por este fenómeno ocurre de forma súbita y rara vez se presenta de manera lenta y progresiva ${ }^{15,16}$. Si bien este factor pudiera explicar algunas parálisis de brusca instalación ${ }^{17}$, debe recordarse que las funciones del nervio se afectan tras bloquear el $50 \%$ de las fibras ${ }^{18}$, y en estudios se ha demostrado que se requieren presiones por sobre la arterial sistólica para producir compresión neural y finalmente parálisis facial, por lo que no sería el principal mecanismo involucrado.

\section{Inflamación}

La exposición directa del nervio facial a fenómenos inflamatorios podría producir cambios degenerativos, caracterizados por desmielinización segmentaria e hipertrofia de las células de Schwann. Estos cambios se han demostrado en pacientes con otitis media crónica, aun sin presentar parálisis facial ${ }^{19}$. Los mastocitos, elevados en algunas neuropatías periféricas ${ }^{20}$, podrían actuar mediante sus proteasas para producir los focos de desmielinización $n^{21}$.

\section{Osteítis y destrucción}

En infecciones crónicas del oído medio, la parálisis facial se puede producir por el crecimiento lento y progresivo de un colesteatoma o por ostétis del canal de Falopio. Se plantea en general que una infección ótica persistente puede producir infec- 
ción ósea y resorción con la formación de mastoiditis coalescente que puede evolucionar a osteítis y erosión ósea, la que culmina finalmente en la destrucción del nervio ${ }^{4}$. Debe recordarse que el fenómeno de osté́tis tarda dos a tres semanas en desarrollarse tras el inicio de la infección, por lo que no puede considerarse relacionado al curso de una otitis media aguda.

\section{Infección/Tóxico}

La fisiopatología por la cual una otitis media aguda produce parálisis facial precoz es desconocida. Estudios bacteriológicos aún no son concluyentes sobre la posibilidad de que toxinas bacterianas sean responsables de la parálisis facial, dado que no se han demostrado este tipo de toxinas en las bacterias patógenas del oído medio ${ }^{4}$. Sin embargo, la relación temporal existente entre otitis media aguda y parálisis facial sugiere la presencia de mediadores que pueden actuar directamente como neurotoxinas ${ }^{18} \mathrm{O}$ indirectamente reactivando virus latentes, como por ejemplo el virus herpes zoster y virus herpes simples, los cuales se han identificado en pacientes afectados con otitis y parálisis facial22-24.

\section{Invasión directa bacteriana}

Lainfección se puede propagar en el canal óseo del facial por dehiscencias y canalículos fisiológi$\cos ^{1,25}$. Debe recordarse la alta frecuencia de dehiscencias fisiológicas en el segmento timpánico del nervio facial, que podrían predisponer en algunos pacientes el desarrollo de una parálisis facial9,26. Los canalículos fisiológicos se encuentran en las salidas para los nervio estapedial y cuerda del tímpano. En el caso de la otitis media crónica, los pólipos, el tejido de granulación y/o un colesteatoma pueden erosionar el canal de Falopio con la subsiguiente diseminación de la infección al canal. Dada la alta frecuencia de dehiscencias en el oído medio y la baja aparición de parálisis facial debe considerarse que el nervio presenta una alta resistencia a la infección.

\section{Alteraciones inmunológicas}

Algunas enfermedades inmunológicas tales como sarcoidosis, poliarteritis nodosa y granulomatosis de Wegener pueden producir parálisis facial por medio de vasculitis de la vasa nervorum, neuropatía o compresión ${ }^{27}$, los cuales pueden coexistir con alteraciones de la audición y otros pares craneanos.

En suma, la parálisis facial es una complicación infrecuente de una otitis media, aguda o crónica, su fisiopatología sigue siendo desconocida y habitualmente pueden estar involucrados uno o más factores. En otitis media aguda se plantean como probables mecanismos la invasión bacteriana directa del nervio facial a través de dehiscencias óseas, el edema inflamatorio que produce compresión y trombosis de la vasa nervorum, la reactivación de virus neurotrópicos latentes, la neurotoxicidad que produciría desmielinización o alteraciones inmunológicas del huésped ${ }^{28}$. Con relación a la otitis media crónica, se le considera un proceso evolutivo en el que participan el tejido de granulación, la osteítis y la presencia de un colesteatoma. Usualmente el segmento timpánico del nervio facial es el más frecuentemente afectado (77\%), debido a la presencia de dehiscencias o por destrucción de la pared ósea causada por un colesteatoma (presente en el $75 \%$ de las otitis media crónicas que se complican) ${ }^{29}$.

\section{EVALUACIÓN CLÍNICA}

La evaluación clínica inicial frente a una parálisis facial busca diferenciar entre el origen central o periférico de la lesión. Dentro de los signos más característicos de la periférica se encuentran el compromiso hemifacial (incluyendo el tercio supe rior) y el fenómeno de Bell. Las centrales se caracterizan por mantener movilidad en el tercio superior del rostro, movimientos en reacciones emotivas y no se evidencia compromiso lacrimal o de salivación. 日 diagnóstico diferencial de una parálisis facial es extenso (Tabla 1), por lo que una anamnesis acabada y un examen físico completo son los mejores elementos para determinar la causa, y en base a los hallazgos clínicos decidir qué exámenes solicitar ${ }^{30}$. La valoración de la seve ridad se puede realizar según la escala de HouseBrackmann (Tabla 2).

Cásicamente se han descrito exámenes que pueden ayudar a determinar el nivel de la lesión del 
Tabla 1. Diagnóstico diferencial de parálisis facial periférica

\begin{tabular}{|ll|}
\hline - Traumáticas & Fractura del hueso temporal \\
& Colesteatoma \\
& Neurinoma del acústico \\
& Leucemia \\
& Neoplasias parotídeas \\
& Virus coxsackie, herpes zoster, VIH, influenza, parotiditis \\
& Enfermedad de Kawasaki \\
& Arañazo de gato \\
& Enfermedad de Lyme \\
& Mycoplasma \\
& Mastoiditis, meningitis \\
& Otits externa \\
& Sífilis, tuberculosis \\
& Síndrome de Guillian Barré \\
& Post inmunización \\
& Intoxicación plúmbica \\
& Parálisis de Bell \\
- Idiopáticas & Higroma quístico \\
Congénitas & Quiste branquial \\
& Síndrome de Moebius \\
\end{tabular}

Tabla 2. Escala de House-Brackmann para evaluar severidad de parálisis facial

\begin{tabular}{|ll|}
\hline Grados & Evaluación \\
\hline GRADOI & $\begin{array}{l}\text { Función normal, simétrica en todas las áreas } \\
\text { Leve debilidad evidente en examen meticuloso; cierre palpebral completo con mínimo } \\
\text { esfuerzo, leve asimetría en sonrisa con máximo esfuerzo, sincinesias apenas perceptibles, } \\
\text { sin espasmo/contractura } \\
\text { Debilidad notoria no desfigurante, cierre palpebral completo, puede no elevar el párpado, } \\
\text { asimetría en movimientos de boca en máximo esfuerzo, sincinesias presentes, espasmos } \\
\text { musculares }\end{array}$ \\
GRADOIII & $\begin{array}{l}\text { Asimetría deformante, cierre palpebral incompleto, asimetría en movimientos de boca, } \\
\text { sincinesias severas, espasmos musculares }\end{array}$ \\
GRADOVIV & $\begin{array}{l}\text { Movilidad casi imperceptible, cierre palpebral incompleto, ligero movimiento comisura } \\
\text { labial, sincinesias y espasmos generalmente ausentes }\end{array}$ \\
GRADOVV & Sin movilidad, paridad del tono, sin sincinesias ni espasmos musculares \\
\hline
\end{tabular}

nervio facial en una parálisis facial periférica, los que se basan en la evaluación de la funcionalidad de los ramos emitidos por el nervio facial. De esta forma se han descrito el test de Schirmer que evalúa el nervio petroso superficial mayor, el reflejo estapedial se puede utilizar para el nervio del músculo del estribo y test del sabor para evaluar la cuerda del tímpano. Actualmente los más utilizados son los dos primeros. 1 test de Schirmer evalúa la lacrimación en 5 minutos, considerándose alterada con una asimetría de $25 \%$ o si es menor a $25 \mathrm{~mm}$. 日 reflejo estapedial se basa en la 
contracción muscular que cambia la impedancia de la cadena osicular a determinada intensidad sobre el umbral auditivo. Debe recordarse que estos test pueden no ser concordantes, dado que el daño en el nervio puede abarcar uno o múltiples segmentos.

Los test de electrodiagnóstico buscan determinar el pronóstico de recuperación frente a una parálisis facial completa $\mathrm{日}$ test de excitabilidad, el test de estimulación máxima y la electroneuronografía son los que presentan mayor utilidad. Estos exámenes no deben ser realizados hasta después de tres o cuatro días luego del inicio de la parálisis facial debido a que la degeneración walleriana alcanza el lugar de registro aproximadamente tras 48 a 72 horas de ocurrida la injuria neural ${ }^{31}$. De los test antes mencionados la electroneurografía es la más útil dada su objetividad y estimación cuantitativa de degeneración neural ${ }^{1}$. La electromiografía tiene escaso valor en forma temprana, dado que los potenciales de fibrilación aparecen sólo al décimo día, pero su uso tras la segunda semana de evolución puede demostrar potenciales polifásicos como signo de reinervación $^{32}$.

La relación entre parálisis facial y otitis media requiere un cuadro otológico bien definido al momento del diagnóstico. En este contexto, son los elementos clínicos presentes en la historia y al examen físico, apoyado por otomicroscopía, los necesarios para el diagnóstico. La parálisis facial puede desarrollarse precozmente frente a una otitis media aguda, siendo periférica e ipsilateral al cuadro otológico, generalmente incompleta y alcanzando su máximo en aproximadamente un día. $\mathrm{日}$ lapso de tiempo que transcurre hasta el inicio de la mejoría clínica no es preciso, mientras el tiempo en que se logra la remisión de la sintomatología es variable, siendo mayor para los grados más severos de parálisis sin relación a la duración de la otitis. En otitis media crónica, con mucosa granulomatosa o polipoidea, la parálisis en general es de inicio paulatino y curso progresivo $(75 \%)^{18}$, $50 \%$ sin síntomas asociados y frecuentemente como complicación única ${ }^{29,33}$. Dentro de las características descritas destaca que cerca del $80 \%$ de los pacientes refieren una historia de otitis media crónica con una evolución mayor de 10 años.
Los cultivos bacteriológicos de secreción del oído medio son generalmente negativos ${ }^{3,34,35}$, sin embargo cuando existe un agente, por lo general pertenece al grupo de otopatógenos, de esta forma Streptococcus pneumoniae, Haemophylus influenzae y Moraxella catarrhalis son los más frecuentemente citados ${ }^{36}$.

Frente a una parálisis facial como complicación de una otitis media, los exámenes de laboratorio no son útiles y el rol del electrodiagnóstico no ha sido establecido, ya que sólo permite predecir la función final ante una parálisis completa ${ }^{34}$, pero no influye en la conducta terapéutica inicial ni en la decisión quirúrgica. La tomografía computada de oídos puede ser útil, al entregar adecuada información sobre la presencia de dehiscencias, mastoiditis coalescente, extensión de colesteatoma y la presencia de complicaciones intracraneales asociadas ${ }^{5}$, pero no constituye un requisito indispensable en cada caso (al menos inicialmente).

\section{TRATAMIENTO}

\section{Parálisis facial en otitis media aguda}

Ouando la parálisis facial se presenta en el contexto de una otitis media aguda el manejo debe incluir una terapia antibiótica agresiva²2. La mayoría de los autores coinciden en que el tratamiento de elección es el inicio de antibioterapia endovenosa, orientada a la microbiología, asociada a miringotomía amplia, con instalación de tubo de ventilación $n^{1,5,34}$. En el caso que los síntomas no mejoren se debiera realizar una tomografía computada de oídos para descartar la presencia de un tumor oculto o el desarrollo de una mastoiditis coalescente ${ }^{1,22}$.

日 uso de corticoides es controversial, porque mientras algunos autores apoyan su uso en procesos inflamatorios del oído medio ${ }^{22}$, otros lo omiten dado sus eventuales complicaciones. Existe escasa experiencia con respecto al uso de antivirales, como el aciclovir, que se basa principalmente en las experiencias de tratamiento de la parálisis de Bell y el herpes zoster ${ }^{24,37}$. No se debe olvidar el cuidado ocular en estos pacientes, mediante el uso de lágrimas artificiales y/o sello ocular.

$\boxminus$ acto quirúrgico estaría indicado si se evidencia un compromiso destructivo de la mastoides ${ }^{36}$, 
frente al cual se aconseja realizar una mastoidectomía sin descompresión del nervio facial, dado un alto riesgo de daño en este tipo de casos $^{22}$. En las series en que se han realizado descompresión del nervio facial se describen peores resultados al abrir el epineuro que sin incindirlo ${ }^{34,38}$. Otras indicaciones de tratamiento quirúrgico son: frente a una alta sospecha de enfermedad fúngica, tuberculosis, neoplasias, granuloma eosinofílico o alteraciones autoinmunes ${ }^{18}$.

\section{Parálisis facial en otitis media crónica}

日 tratamiento consiste en remover la infección y descomprimir el nervio facial en forma urgente. Cuando la causa es una otitis media crónica colesteatomatosa la indicación quirúrgica es absoluta siendo las alternativas múltiples según la gravedad y extensión de las lesiones.

En la mayoría de los casos se trata de amplios colesteatomas que destruyen por completo la caja del tímpano, al antro y la mastoides, y que finalmente destruyen en mayor o menor medida el canal óseo del nervio facial. En tales casos se realiza una cirugía radical de oído medio y una descompresión quirúrgica del nervio facial, desde el ganglio geniculado al agujero estilomastoideo, recomendándose no incindir el epineuro, salvo que exista un compromiso evidente de éste ${ }^{33,39,40}$.

En casos de lesiones más circunscritas se realiza la exéresis de la lesión acompañada de descompresión del nervio facial, pero se debe intentar conservar parte o la totalidad de la cadena osicular, pudiendo incluso realizar reconstrucción de cadena en el mismo tiempo quirúrgico.

\section{PRONÓSTICO}

En general el pronóstico de recuperación en el caso de una parálisis facial con relación a una otitis media aguda es bueno, y se puede esperar la regeneración completa del nervio ${ }^{3,22,28}$. La mayoría de los pacientes tienen una función muscular completa de tres a seis semanas de evolución; sin embargo en un pequeño porcentaje, y a pesar del inicio precoz del tratamiento, la parálisis facial puede ser permanente por regeneración anómala del séptimo par. Las complicaciones más frecuen- tes de la regeneración anómala incluyen sincinesias (movimientos involuntarios asociados a movimientos voluntarios), lagrimeo (lágrimas de cocodrilo) y espasmos hemifaciales.

En el caso de la otitis media crónica el pronóstico de mejoría bordea el $60 \%$ a $70 \%^{29,33}$, sin diferencias según hallazgos quirúrgicos, la presencia de colesteatoma ni por técnica quirúrgica utilizada. 日 impacto de la edad y el retraso en la decisión quirúrgica en el pronóstico de la parálisis aún no están completamente dilucidados ${ }^{41}$.

\section{BIBLIOGRAFÍA}

1. Gaio E, marioni G, de flippis C, tregnagh A, Caltran S, Starteril A. Facial nerve paralysis secondary to acute otitis media in infants and children. J Paediatr Child Health 2004; 40: 4836.

2. Kangsanarak J, FoOANant S, RugkphaOpunt K, Navacharoen N, Teotrakul S. Extracranial and intracranial complications of suppurative otitis media. Report of 102 cases. J Laryngol Otol 1993; 107(11): 999-1004.

3. Elefen B, Bonding P. Facial palsy in acute otitis media Cin Otolaryngol Allied Sci 1996; 21 : 393-5.

4. Polook RA, Brown LA. Facial paralysis in otitis media. In Graham MD, House WF, editors. Disorders of the facial nerve. New York: Raven; 1982. p. 221-4.

5. WHITE N, MoCans KM. Facial paralysis secondary to acute otitis media. Pediatr Emerg Care 16(5): 343-5.

6. HaRVEY S, Fox M. Relevant issues in revision and canal-wall-down mastoidectomy. Aolaryngol Head Neck Surg 1999; 121(1): 18-22.

7. SeESNIOK SH, LyNN-MACRAE AG The incidence of facial nerve dehiscence at surgery for cholesteatoma. Otol Neurotol 2001; 22: 12932.

8. Weling DB, Gassoock ME, Gantz BJ. Avulsion of the anomalous facial nerve at stapedectomy. Laryngoscope 1992; 102(3): 729-33.

9. BAXTER A. Dehiscences of the fallopian canal. $J$ Laryngol Ool 1971; 85: 587-594.

10. Moreano 日u, Paparela MM, Zhterman D, GorcooleA MV. Prevalence of facial canal 
dehiscence and of persistent stapedial artery in the human middle ear: a report of 1,000 temporal bones. Laryngoscope 1994; 104(3 Pt 1): 309-20.

11. Di Martino E, Selmaus B, Haense J, Sahlege JG, Westhofan M, Prescher A. Fallopian canal dehiscences: a survey of clinical and anatomical findings. Eur Arch Otorhinolaryngol 2005; 262(2): 120-6.

12. Barnes G, liang JN, Michaes L, Wright A, Hall S, GEESon M. Development of the fallopian canal in humans: a morphologic and radiologic study. Ool Neurotol 2001; 22(6): 931-7.

13. Dugic D, SAvic D. Otogenic facial paralysis. A histopathological study. Eur Arch Oorhinolaryngol 1990; 247(3): 143-6.

14. Saito H, TAKBDA T, Kishimoto S. Facial nerve to facial canal cros-sectional area ratio in children. Laryngoscope 1992; 102(10): 11726.

15. Devriese PP. Compression and ischaemia of the facial nerve. Acta Otolaryngol (Stockh) 1974; 77(1): 108-18.

16. TZadiK A, Babin RW, RYu JH. Hypotensioninduced neuropraxia in the cat facial nerve. Otolaryngol Head Neck Surg 1982; 90(2): 1637.

17. Fsch U, Feıx H. On the pathogenesis of Bell's palsy. Acta Otolaryngol (Stockh) 1983; 95(5-6): 532-8.

18. Elıот CA, ZALAL GH, GoTtLI円B WR. Acute otitis media and facial paralysis in children. Ann Otol Rhinol Laryngol 1996; 105(1): 58-62.

19. DueRIC D. Neuropathy of the facial nerve in chronic otitis media without associated facial paralysis. Eur Arch Atorhinolaryngol 1990; 247(4): 232-6.

20. Osson Y. Mast cells in human peripheral nerves. Acta Neurol Scand 1971; 47(3): 35768.

21. Johnson $D, S$ m mast cells in demyelination. I. Myelic proteins are degraded by mast cell proteases and myelin basic protein and P2 can stimulate mast cell degranulation. Brain Res 1988; 444(1): 195-8.

22. JosfrH E, SPgRING N. Facial nerve paralysis in acute otitis media: cause and management revisited. Otolaryngol Head Neck Surg 1998; 118(5): 694-6.
23. Sugta T, Murakami S, Yanagihara N, Fuimara Y, HIRATA Y, KURATA T. Facial nerve paralysis induced by herpes simplex virus in mice: an animal model of acute and transient facial paralysis. Ann Otol Rhinol Laryngol 1995; 104: 574-81.

24. StafFord FW, Wear AR. The use of Acyclovir in Ramsay-Hunt syndrome. J Laryngol Ool 1986; 100(3): 337-40.

25. Kamitsuka M, Fegdman K, Richardson M. Facial paralysis associated with otitis media. Pediatr Infect Dis 1985; 4(6): 682-4.

26. Candea FA, Stemart TJ. The pathophysiology of otologic facial paralysis. Otolaryngol Cin North Am 1974; 7: 309-30.

27. Drinias V, Florentzson R. Facial Palsy and Wegener's granulomatosis. Am J Otolaryngol 2004; 25(3): 208-12.

28. Redaeli de Znis LO, Gamba P, Balzaneli C. Acute otitis media and facial nerve paralysis in Adults. Otol Neurotol 2003; 24(1): 113-7.

29. SAvic DL, DJøIC DR. Facial paralysis in chronic suppurative otitis media. Cin Otolaryngol Allied Sci 1989; 14(6): 515-7.

30. Eshe G, LaHAt E, AzIZ E Peripheral facial nerve paralysis in children: The value of routine diagnostic work-up studies. Cin Neurol Neurosurg 1988; 90(4): 339-42.

31. KNox GW. Treatment controversies in Bell palsy. Arch Otolaryngol Head Neck Surg 1998; 124(7): 821-3.

32. OHANG CY, CASS SP. Management of facial nerve injury due to temporal bone trauma. Am J Otol 1999; 20(1): 96-114.

33. Altuntas A, Unal A, Aslan A, Ozcan M, KuRKOUOGLU S, NALCA Y. Facial nerve paralysis in chronic suppurative otitis media: Ankara Numune hospital experience. Auris Nasus Larynx 1998; 25(2): 169-72.

34. Goldsten NA, Cassebrant ML, Bluestone CD. Intratemporal complications of acute otitis me dia in infants and children. Otolaryngol. Head Neck Surg 1998; 119(5): 444-54.

35. Vassbotn FS, Klausen OG, Lind O, Molla P. Acute mastoiditis in a Norwegian population: a 20 year retrospective study. Int $J$ Ped Otorhinolaryngol 2002; 62(3): 237-42.

36. Zapalac JS, Biluings KR, Sommade ND, Roland PS. Suppurative complications of acute otitis media 
in the era of antibiotic resistance. Arch Aolaryngol Head Neck Surg 2002; 128(6): 660-3.

37. ADOUR KK, RUBOYIANES JM. Bell's palsy treatment with acyclovir and prednisone compared with prednisone alone: a double-blind, randomized, controlled trial. Ann Otol Rhinol Laryngol 1996; 105(5): 371-8.

38. Teisch F, Ohandlg JR, May M et al. Infection: otitis media, cholesteatoma, necrotizing external otitis, and other inflammatory disorders. In: May M, Schaitkin BM, eds. The Facial Nerve, $2^{\text {nd }}$ ed. New York: Thieme, 2000; 383-92.
39. HaRk $\operatorname{l}$ LA, Pignatari SS. Facial nerve paralysis secondary to chronic otitis. Am J Otology 1992; 13(4): 372-4

40. IKEDA M, NaZAKOTO H, OMODA K, Hiral R, KidA A. Facial nerve paralysis caused by middle ear cholesteatoma and effects of surgical intervention. Acta Otolaryngol 2006; 126(1): 95-100.

41. Yemisgr $S$, Tosun F, Kazkayasi M. Facial nerve paralysis due to chronic otitis media Atol Neurotol 2002; 23(4): 580-8. 